PRE-PRINT Version (September 2010)

Submitted to HICSS-44 (Hawaii International Conference on System Sciences), 2011.

\title{
Democracy.com: A Tale of Political Blogs and Content
}

\author{
Karine Barzilai-Nahon \\ University of Washington \\ karineb@uw.edu
}

\begin{abstract}
The debate about the role of political blogs in politics generally and its effect on democracy and participation in particular has deepened since the 2008 U.S. presidential election. While some studies warn that the Internet may undermine deliberation, and replicate patterns of homophily and polarization among blogs with the same political inclination, other studies emphasize the potential of the Internet to strengthen cross-ideological discourse and participation. This paper suggests, using a hybrid theoretical framework which acknowledges homophily and the power law distribution among political blogs, and at the same time exhibits the use of the Internet also as a cross-participation platform and as strengthening participation. For that purpose, this paper looks at 83 videos that went viral during the 2008 election and examines patterns of behavior of the top 50 political blogs (conservative and liberal) in respect these videos over a period of two years.
\end{abstract}

\section{Introduction}

The Internet played a critical role in the 2008 Presidential Election. The use of the Internet for political purposes has grown dramatically (74\% of internet users in the U.S. visited blogs for political information). Political blogs were one of the main information venues of public attention regarding the elections (26\% of internet users) [1]. Also, the 2008 election was characterized by a growing use of social networking applications, including video platforms, which became important vehicles for mobilization by political elites and users [2]. By the end of the campaign, fully $60 \%$ of online political users had watched some sort of video related to the politics of the election [1]. The increasing appropriation of information technologies for political purposes has deepened the debate about the effect of the Internet on politics and on mobilization. Some scholars argue that the Internet increases democratic practices, while

\author{
Jeff Hemsley \\ University of Washington \\ jhemsley@uw.edu
}

other scholars, holding more skeptical views suggest that the Internet may enhance acute polarization, fragmentation and homophily among users.

This paper reports on findings from an empirical study done on 83 videos that went viral during the 2008 U.S. presidential election cycle. Our paper examines the behavior of 50 top-blogs (conservative and liberal) with respect to how they linked to these election related viral-content between March 2007 and June 2009. Specifically, we examine structures of behavior of political blogs in reference to content.

Our paper is structured as follows: First, we provide a literature review about the debate on the role of political blogs on political participation and deliberation. We also survey literature which focuses on viral videos and political behaviors of users. Following this we explain our methodology, and how it differs from previous studies, and also locate our contribution within the body of literature. Finally, we present our findings and discuss them.

\section{Two Bodies of Scholarship}

Recently, the debate over the role of the Internet in general and political blogs in particular, within the societal-political ecological system has increased. The focus of the debate has been around the question of how the internet affects politics in general and democratic practices in particular. Cass Sunstein raised normative concerns regarding fragmentation [3] and polarization [4] of content caused by information technologies. His main argument was that information technologies, while providing historically unprecedented diversity of freedom of expression, also provide self-regulating mechanisms that allow users to customize content feeds such that, people will tend to read information that is aligned with their basic assumptions. For Sunstein, this may lead to a cyber-balkanization of the Internet, fragmenting users into narrowly-focused groups who 
share similar opinions and are only exposed to information that confirms their previously-held opinions.

Some empirical studies have reinforced some aspects of this normative view by finding patterns of political homophily between top blogs, that is, there is a tendency of blogs with the same political and ideological inclination to link to each other [5][6]; or tendency of blog readers to read blogs aligned with their ideological and partisan preferences [7]. Other studies have also shown patterns of polarization [7][8]. Joining this stream of research, another line of research has emphasized the power law distribution of actors in networks [9] and particularly of political blogs [5][10][11]. The claim is for the existence of a skewed distribution in the blogosphere, where top blogs capture the majority of attention from mainstream media elites and readers, and receive a disproportionately large number of links compared to other blogs. In other words, nodes that are rich in ties are likely to become even richer over time. Farrel and Drezner also claimed that the top blogs influence political elites through media actors who read them frequently [10] and act as network gatekeepers [12].

The combination of these two streams of research, where political blogs exhibit homophily and polarization, and that the blogosphere follows a power-law distribution are key elements in the debate on the influence of blogs on the public sphere, on public opinions, participation and deliberation. Some have argued that the exhibited polarization and homophily, with a lack of dialogue, is a recipe for little deliberation. Also for these scholars, the power law distribution of blogs means that the Internet does not manifest a pluralized space as people had hoped, and instead reflects a problem of participation [8].

However, other scholars have offered an opposing view claiming that the Internet has increased the available spectrum of choices and therefore provides users with a growing ability to participate. More particularly, Benkler and Shaw [13] and Woodly [14] argue that blogs empower the public and enhance deliberative democracy. While acknowledging the homophily, reported in so many empirical studies, Benkler and Shaw argue that "the foundational question for a wider range of democratic theories is who has the opportunity to be heard at all, and to form a sufficient level of coherence around an issue to turn it into a credible item on a society's political agenda" [13]. They criticize the body of literature that claims the existence of polarization through link analysis of political blogs, by emphasizing limitations of these methodologies: for example, underestimating the size of authors of blogs, structure of the blog or features provided to users. Instead, Benkler and Shaw suggest studying opportunities for discursive practices by using qualitative methodologies to capture factors such as: opportunities that support user-generated content, mobilization through calls for action and content authorship and style.

Both sets of literature address similar questions of users' participation and of understanding the effect of information technology on politics. Both sets of literature also look at the same 'end product'. That is, whether the Internet increases or decreases discursive democracy, or whether information technologies fragments or diversify information. But these sets of literature use different lenses and while they both examine the 'end product', sometimes they talk across each other.

The underlying concern of both sides has to do with determining the conditions that are sufficient for creating, or maintaining, stable democratic practices, and they examine what exists today. One perspective claims that providing technology that enables a larger space of choices and a growing capacity to be heard is sufficient. Benkler and Shaw state that, "the creation of such pathways improves the openness of the public sphere to views and agenda-setting efforts outside the traditional sources of discursive and cultural power" [13]. However, others require the exercise and translation of these choices into actions. In other words, one side contends that by providing information technologies that enable debate, we have sufficient conditions for stable democracy, and the other side, that those information technologies need to actually be used.

In terms of methodologies, most empirical studies that focus on the technology as an enabler of choices focus on studying the structure and design structure of the technology [13][15]. Thus, the main focus of these studies is on the perceived value of information and how technology may be used or is used in particular contexts. While studies emphasizing polarization, homophily and the power law distribution focus on measuring the holistic patterns of behavior which still show unequal and skewed distributions of power [2]. Benkler and Shaw point out that most of these studies use link analysis as a method. While link analysis may have its flaws, as Benkler and other suggest [13], these patterns have been supported through other methods (i.e., content analysis and surveys) [6][7][1]. 
So, while we perceive a glass-half-full vs. glasshalf-empty split in the literature, we note that at their core, both streams of research are fundamentally interested in illuminating and discussing conditions that support stable democratic practices. We argue for a hybrid theoretical framework, that strives to achieve an empirical synthesis acknowledging the power-law distribution of political blogs and homophily while at the same time pointing to an increasing pattern of behavior of participation and cross-ideological discussions. Thus we merge these two streams, embracing some of the main arguments from both perspectives. Recently a few studies have demonstrated this approach [16][6][7]. For example, Hargittai et al. notes that while political elites on the Internet may capture most of the attention, users may shift attention and be active in discourse, thus increasing practices of democratic behaviors [17].

\section{Viral Videos and Political Blogs}

Literature about political blogs and their impact on political agenda setting and decision makers is mixed. Klotz [18] studied the 2008 Senate campaign season, and found that videos produced by ordinary citizens were generally undistinguished and over shadowed by videos created by institutional participants. However, Wallsten, looking at the most viral video of the 2008 election, "Yes We Can", a song created by the singer will.i.am, argues that bloggers and members of the Obama campaign were significant factors in driving viewership of the video, while journalists appeared to have little or no impact on viewership or blog discussion [19]. This contradicts Farrell and Drezner's argument that although blogs have low viewership, nevertheless they are being read frequently by journalists and media elites, which brings them to the attention of the public and to the political arena [10]. Karpf supports this claim and argues that web 2.0 technologies may be making elite networks more porous by bringing content that would otherwise remain obscure into the public eye [20].

Furthermore, most literature is reporting on higher volumes of user-generated content by liberals. Klotz [18] shows that a majority of the production of YouTube videos was created by left-leaning produces. This is supported also by Robertson et al. [16] and by Smith [1] who found higher levels of activity in terms of producing content and linking patterns of liberals over conservatives. It stills needs to be determined whether the advantage in message diffusion can be translated into actual political mobilization, or to higher levels of deliberation.
This paper takes a hybrid perspective in two ways; first, by looking through social network analysis at how top-blogs link, and thus refer users, to viral video content, which is fundamentally different than a blog-to-blog link analysis, and second, we explore the nature of the content that has been linked to by both liberals and conservatives at the time of the election. Thus, the contribution of this paper is as follows: 1) we show there are differences and similarities in the ways that blogs of different ideological leaning link to external content, which we believe reflects their approaches to message diffusion, 2) we specifically focus on viral videos linked to from top-blogs during the 2008 presidential election cycle, 3) we take a hybrid-approach, using link analysis to illuminate content linking behavior at a macro level, and then analyze specific examples of content that spans the ideological divide.

\section{Research Questions and Methodology}

This paper attempts to address a gap in the literature about the way in which political blogs link to content. Specifically, the content, that we consider, are videos that went viral during the 2008 US presidential election. Our hope is to illuminate structures of behavior of political blogs in reference to content. To that end, we address the following research questions: 1 . Do political blogs of the same political inclination tend to link to the same content? 2. Do political blogs follow a bandwagon effect, that is, is there a positive relationship between the likelihood of a blog linking to content and the popularity of that content? 3 . Are blogs with a higher number of comments more likely to refer to viral videos?

We are using quantitative analysis which includes descriptive statistics as well as social network analysis (heretofore: SNA) as a means of addressing these questions. Numerous authors have noted the limitations of quantitative link analysis [21][22][13], mainly being that it fails to answer 'why' questions, is unable to provide a qualitative insights of the links, and cannot identify or describe the context within which the links exist. Therefore, we are using mixed methods in order to address our research questions and minimize these limitations. Through this methodology: 1) we hope to understand general, macro-level patterns that reflect theories as discussed above, 2) SNA allows hypothesis testing, which gives us a certain level of confidence that these trends are not random, 3) our efforts extend existing work by looking at blog-to-content links instead of blog-to- 
blog links, which allow us to unveil patterns previously overlooked.

Since we are looking at political blogs linking to content (specifically, videos), we necessarily collect data that represents those links. We aimed to collect data which represents the videos that went viral during the 2008 election, as well as data about the blogs that linked to those viral videos. We used the following methodology in collecting that data. The set of videos was drawn from ViralVideoChart.com on January 20th, 2009. We drew the top 100 videos over the preceding year in the following categories: over all Top videos, top 100 political videos, and top 100 election videos. The lists were combined and any redundancy was removed. Four researchers (a faculty member and three PhD students) coded the videos as related to the election by answering the question: "Was the content related to the 2008 presidential campaign?” For a video not to be included, all four had to agree that it was not related to the election. The result was 120 videos. Next we gathered daily views data for these viral videos. This was done through TubeMogul. We were able to gather daily view data for 83 videos that spanned from March 2007-June 2009.

Our next goal was to identify blogs related to these videos. For that purpose, we used the YouTube unique identifier (i.e., a unique video URL) for each one of the 120 viral videos identified in the previous stage. We created scripts which harvested automatically all of the blogs (not only top-blogs) linking to these viral videos on a daily basis. The scripts harvested the list of blogs through two blog search tools: Technorati and Google Blog Search. These searches gave us a dataset of over 10,000 blogs linking to these viral videos during March 2007 and June 2009. Since for this study, we are primarily focused on top-blogs, we also used a third source to collect this list.

Our set of top-blogs was drawn from David Karpf's Blogosphere Authority Index (BAI) [23][11], which is one measure of a blogs authority. Note that the rankings of blogs may change from week to week. Our set contains the top 25 conservative, and top 25 liberal blogs from the week of August 8th of 2008 (see table 1). Note also, that some of the blogs contained in the BAI list do not link to any video in our dataset. The next step was to create a matrix of our top-blogs that linked to our set of viral videos. The result is a matrix with 50 blogs and 83 videos.
Table 1 - Top Political Blogs

\begin{tabular}{|l|l|l|}
\hline & Liberal Top-Blogs & Conservative Top-Blogs \\
\hline $\mathbf{1}$ & DailyKos & Hot Air \\
\hline $\mathbf{2}$ & Huffington Post & Michelle Malkin \\
\hline $\mathbf{3}$ & Think Progress & Ace Of Spades HQ \\
\hline $\mathbf{4}$ & Talking Points Memo & Instapundit \\
\hline $\mathbf{5}$ & Firedoglake & Little Green Footballs \\
\hline $\mathbf{6}$ & Atrios & American Thinker \\
\hline $\mathbf{7}$ & Crooks And Liars & Gateway Pundit \\
\hline $\mathbf{8}$ & Washington Monthly & Newsbusters \\
\hline $\mathbf{9}$ & Pandagon & Jihad Watch \\
\hline $\mathbf{1 0}$ & America Blog & Powerline Blog \\
\hline $\mathbf{1 1}$ & Five Thirty Eight & Volokh Conspiracy \\
\hline $\mathbf{1 2}$ & Glen Greenwald & Ann Althouse \\
\hline $\mathbf{1 3}$ & Digby & Jawa Report \\
\hline $\mathbf{1 4}$ & Feministing & Wizbang Blog \\
\hline $\mathbf{1 5}$ & Juan Cole & Outside The Beltway \\
\hline $\mathbf{1 6}$ & The Moderate Voice & Patterico's Pontifications \\
\hline $\mathbf{1 7}$ & Truthdig & Right Wing News \\
\hline $\mathbf{1 8}$ & Open Left & Tim Blair \\
\hline $\mathbf{1 9}$ & Talk Left & IMAO \\
\hline $\mathbf{2 0}$ & Shakespeares Sister & Townhall \\
\hline $\mathbf{2 1}$ & Sadly, No! & Red State \\
\hline $\mathbf{2 2}$ & MyDD & Anti-Idiotarian \\
\hline $\mathbf{2 3}$ & Ezra Klein & QandO \\
\hline $\mathbf{2 4}$ & MattYGlesias & PoliPundit \\
\hline $\mathbf{2 5}$ & Crooked Timber & Belmont Club \\
\hline & & \\
\hline
\end{tabular}

Our model (see figure 1) reflects our three research questions. Each independent variable is derived from one of those questions and is represented by a matrix. In the next paragraphs we will explain in detail each matrix.

Figure 1: our model

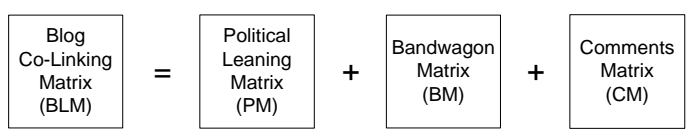

Blog Co-Linking Matrix (BLM). Our dependent variable reflects cases when two blogs link to a video. Thus, we have a bi-partite matrix, which means, it has two types of actors: in our case, blogs and videos, such that we have a 50 blogs-by-83 videos. The data in the cells of the matrix reflect the number of times a blog links to each video. For the purposes of our paper, we are interested in cases where two blogs link to the same video. Therefore, we created a new matrix by multiplying the transpose of the matrix by itself. The cells in the new matrix represent the number of times two blogs link to the same video (which is represented by 28-by-28 matrix).

Political Leaning Matrix (PM). This matrix captures the case where two blogs have the same 
political inclination (conservative or liberal). A cell has a value of one if two blogs share the same political inclination, otherwise the value is zero. We coded the political inclination list by using Dave Karpf BAI (Blogosphere Authority Index) list [23][11].

Bandwagon Matrix (BM). This matrix captures the combined number of views for videos that two blogs linked to. It allows us to test whether or not there is a positive relationship between the number of views of a video and the likelihood of other blogs also linking to that video. In other words, the Bandwagon effect reflects the relationship between the popularity of a video and the tendency of the community to follow that video as well.

Comments Matrix (CM). This matrix allows us to associate the number of comments for a given blog with how it affects the number of times that blog links to a video. For this purpose we are using the Community Activity Score (CAS) of Dave Karpf [23][11].

Each of our variables (BLM, PM, BM and CM) is an adjacency matrix. Thus, we can translate our questions in terms of how the values in one matrix might affect the number of times co-linking occurs between blogs to content in the BLM (our dependent variable). Given this, an appropriate statistical method for our hypothesis tests is network regression [24][25]. The model (figure 1) can be written mathematically as:

$$
E Y_{i j}=\beta_{0}+\beta_{1} x_{1 i j}+\beta_{2} x_{2 i j}+\beta_{3} x_{3 i j}+\varepsilon_{i j}
$$

All of the analysis is carried out using $\mathrm{R}$, which is a powerful open source analysis tool.

\section{Findings}

Similar to other studies [13][1][18], liberals generated higher volumes of content compared to conservatives at the time of the election. Here it is reflected with a higher number of links to videos, 251, compared with 51 links to videos from conservative blogs (see table 2). Top-political liberal blogs were also more active than conservatives in the life cycle of viral videos. Only 10 out of the 25 toppolitical conservative blogs linked to viral videos compared with 18 liberal blogs. Another important finding is that $24 \%$ (20 videos) of all viral videos were not linked at all from any top-blog, conservative or liberal. Since we know that these videos became viral, the above indications may imply a greater role of tail-blogs (blogs of users without high authority) in distributing and sparking an information virality process.

Table 2: Descriptive Statistics

\begin{tabular}{|l|c|c|c|}
\hline LINKS & Value & All & \\
\hline Total links to videos & 302 & $100 \%$ & \\
\hline From Liberal Blogs & 251 & $83 \%$ & \\
\hline From Conservative Blogs & 51 & $17 \%$ & \\
\hline & & & \\
\hline $\begin{array}{l}\text { VIDEOS (linked from } \\
\text { blogs) }\end{array}$ & Value & All & $\begin{array}{c}\text { \% of } \\
\text { linked }\end{array}$ \\
\hline Total Videos & 83 & $100 \%$ & \\
\hline Cross linking Videos & 15 & $18 \%$ & $24 \%$ \\
\hline $\begin{array}{l}\text { Videos Receiving no } \\
\text { links }\end{array}$ & 20 & $24 \%$ & n/a \\
\hline Liberal Only Videos & 45 & $54 \%$ & $71 \%$ \\
\hline All Liberal & 60 & $72 \%$ & $95 \%$ \\
\hline $\begin{array}{l}\text { Conservative Only } \\
\text { Videos }\end{array}$ & 3 & $4 \%$ & $5 \%$ \\
\hline All Conservative & 18 & $22 \%$ & $29 \%$ \\
\hline & 18 & $36 \%$ & $72 \%$ \\
\hline $\begin{array}{l}\text { BLOGS (linking to } \\
\text { videos) }\end{array}$ & Value & All & $\begin{array}{c}\text { \% of } \\
\text { L-C* }\end{array}$ \\
\hline Total Blogs & 50 & $100 \%$ & 25 \\
\hline Liberal Blogs & 10 & $20 \%$ & $40 \%$ \\
\hline Conservative Blogs & $56 \%$ & \\
\hline All & 28 & \\
\hline
\end{tabular}

${ }^{*} \mathrm{~L}-\mathrm{C}$ refers to the conservative or liberal top blogs

Table 3: The Model

\begin{tabular}{|l|l|}
\hline Dependent Variable & Coefficient \\
\hline intercept & 0.21099 \\
\hline Political Leaning & $0.63425^{* *}$ \\
\hline Bandwagon Effect & $0.00756^{* * *}$ \\
\hline Comments & -0.01777 \\
\hline \multicolumn{2}{|c|}{ sig $<.05,{ }^{* *}$ sig $<.01,{ }^{* * *}$ sig $<.001$} \\
\hline Model Performance & \\
\hline Residual standard error & $3.475, \mathrm{df}=1221$ \\
\hline R-squared & 0.7867 \\
\hline F-statistic & $\begin{array}{l}1502, \mathrm{df}=3,1221, \mathrm{p}- \\
\text { value }>.001\end{array}$ \\
\hline
\end{tabular}

The results show that the model explains $79 \%$ of the variance and fits the data well. Moreover, it appears to be statistically significant (see table 3). 


\subsection{Homophily and Cross-references of Political Blogs}

We find support for the idea that blogs of similar political inclination will tend to link to the same videos. We expect the value of an edge to be higher by 0.63 for blogs of the same leaning, than it is for blogs of opposing ideology. In other words, on average, the chance of two blogs linking to a video within the same political leaning is 0.63 higher than between political leanings. Patterns of homophily and fragmentation are also shown through the large number of viral videos that act as silos. $71 \%$ of the viral videos are linked to only by liberals, $5 \%$ are linked to only by conservative blogs and only $24 \%$ of videos are crossed-linked to both by conservative and liberal blogs (we will elaborate more on the cross links later). This suggests a stronger homophily of liberal content than conservative.

Homophily was reported in various studies on the political blogosphere [6][5][7][13]. However, our findings provide an alternative perspective than previously demonstrated because we give a deeper look at how political blogs refer to content, rather than looking at blog-to-blog linking[6][5]. Also, we analyze actual behavior of blogs and users as opposed to their perceived behavior [7][13].

Figure 3 plots the geo-distance of the top-political blogosphere. In other words, it clusters political blogs that have more similar content choices. Nodes that are farther apart in the graph are also farther apart in terms of content choices. Figure 3 emphasizes three main things: first, we can see that there are more liberal blogs (blue) and that liberal blogs are much more active than conservative blogs (as mentioned above). Second, it shows that liberals tend to be more focused around the same content, and tend to be less connected to content that is cross-ideological. Third, it illuminates a new group in the liberal blogs which are more prominent. These can be called the elite of the elite (this supports Dave Karpf argument [23]): a few blogs, like Daily Kos and Huffington Post, that not only attract a lot of traffic, but also draw around them other blogs by deciding and setting the agenda in terms of content, in our case viral videos. We will discuss this more in figure 4 and 5 .
Figure 3: The Geo-Distance of two Blogospheres

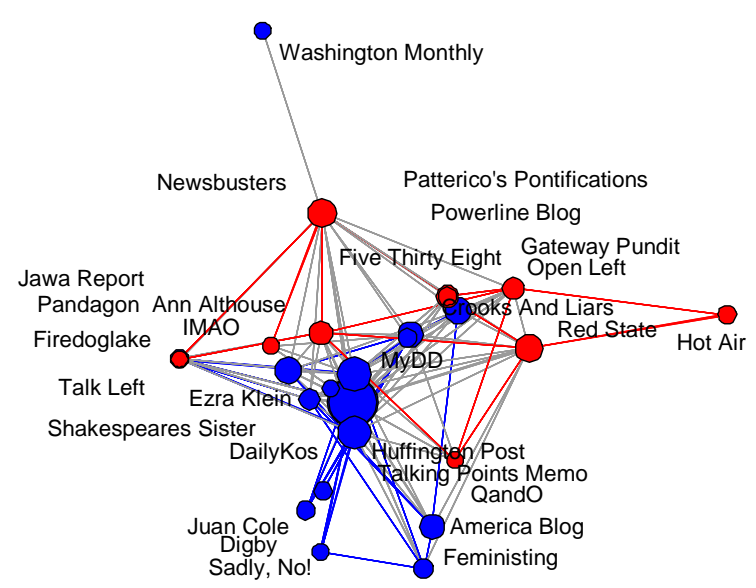

Next, we mapped all of the 15 viral videos that were cross-linked from both conservative and liberal blogs (see figure 4). While cross-linked videos are a minority among the videos (only $18 \%$ of all videos), it is interesting to focus on some of their characteristics, since they reflect content which may serve as a focal point for deliberation and discourse among people with different view point.

Figure 4: Cross Linked Videos

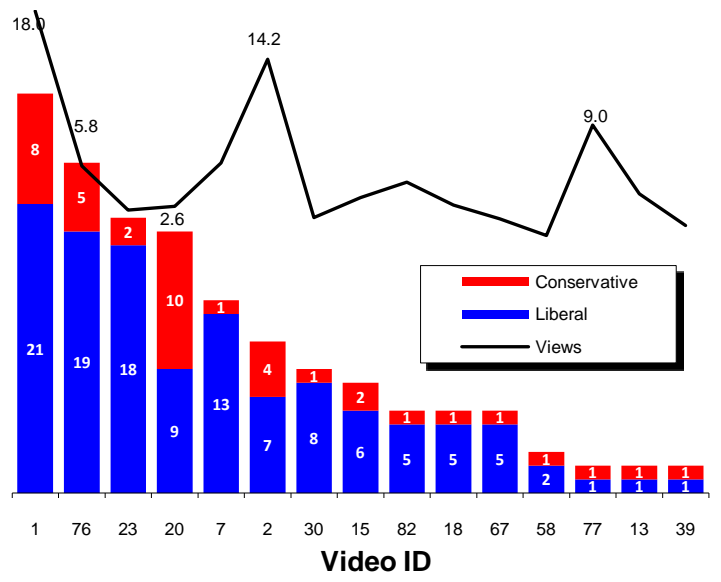

Figure 4 shows the linking distribution between conservative and liberal blogs in the 15 viral videos that were cross-linked. It displays, again, a higher level of blog activity for liberal blogs compared with conservative blogs. Figure 4 also presents the disproportionate production of content by liberals as compared with conservative. Most of the videos that became viral at the time of the election, according to figure 4 and table 1 , are produced by liberals. This is supported in other papers [13][1]. When analyzing the four videos which received the highest attention from the conservative blogosphere, we find that they 
had a common denominator. They had a message or some other audio-video element that could be easily carried and mashed up later to appeal to both sides. An example of a message that can appeal to both sides is in the video "vote differently". Although the video was created by Obama supporters, the message can also fit the agenda of conservative voters. Another video with a cross-message is "Obama Bombshell Redistribution of Wealth Audio Uncovered". These statements of Obama can be used by both sides to emphasize their stances. The other two viral videos that had a high number of cross-links from conservatives were both songs that became popular. Although supported by liberals, voting for Obama, it attracted a larger audience by focusing on the entertainment element of the song. For example, the video "Yes We Can, Obama Song by Will.I.Am", which received over 18 Millions views and was the most viral video in the 2008 Election, or the song "I've Got a Crush on Obama by Obama Girl" with over 14.1 millions views.

Next, figure 5 gives a holistic view of the political blogosphere with respect to linking to viral videos. It gives a better look at the density of the co-links inside the liberal and conservative blogosphere to content vs. cross-links from both conservative and liberal blogs to content. Figure 5 reinforces the strong impact of two liberal blogs, not only on the "liberal" blogosphere, but also on the "conservative" blogosphere. Huffington Post and Daily Kos are a special case which one needs to discus. $70 \%$ of the viral videos were pointed by Huffington Post and $58 \%$ by Daily Kos. According to our data they are the most active blogs in terms of referring to content that is outside of the blogosphere, and covering politicalrelated content. All other top-political blogs cover only $13 \%$ of the viral videos on average, which is a big difference in terms of potential influence between the two super-elite blogs (Huffington Post and Daily Kos) and the others. Additionally, these blogs are the largest among all the political blogs in terms of monthly traffic in their site [23]. Finally, these two blogs are the most crossed-linked according to our data, which means that they acted more like political news aggregators of information rather than representing one side or another. Dave Karpf, addressed this issue as well [23].

Note also that while MyDD is a smaller political blog according to its authority ranking (see table 1), it is very prominent in linking too many videos (32\%) and it looks like the continuous activity promotes the political power of that blog.
Figure 5 - A holistic view of the political blogosphere linking to Viral Videos

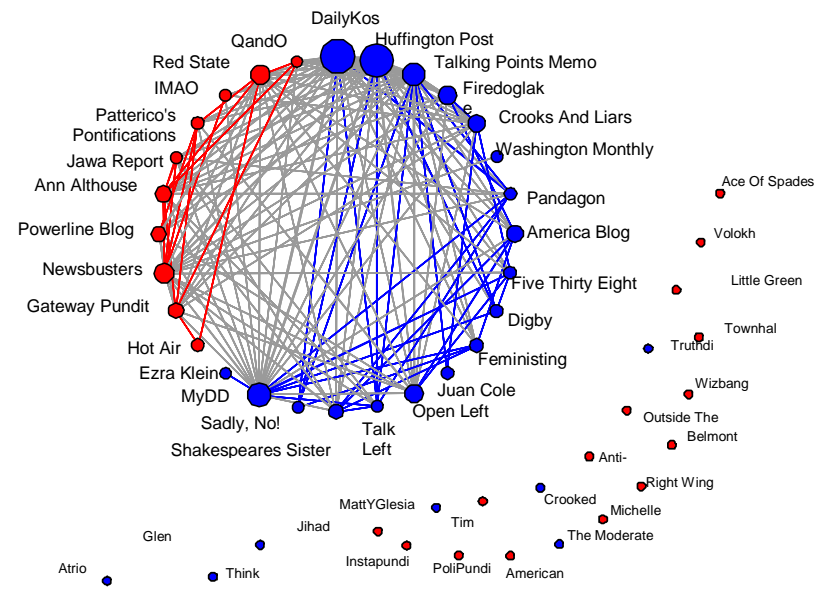

Figure 6 exhibits only the co-linking that occurs to content between liberal-to-liberal blogs and between conservative-to-conservative blogs. Note how the liberal political blog Washington Monthly is not linked and associated with any content of its peers. In fact this blog is cross-linking to content with the conservative blog Newsbaster (see Figure 7).

\section{Figure 6 -Co-linking inside Liberal and Conservative Blogs to Content}

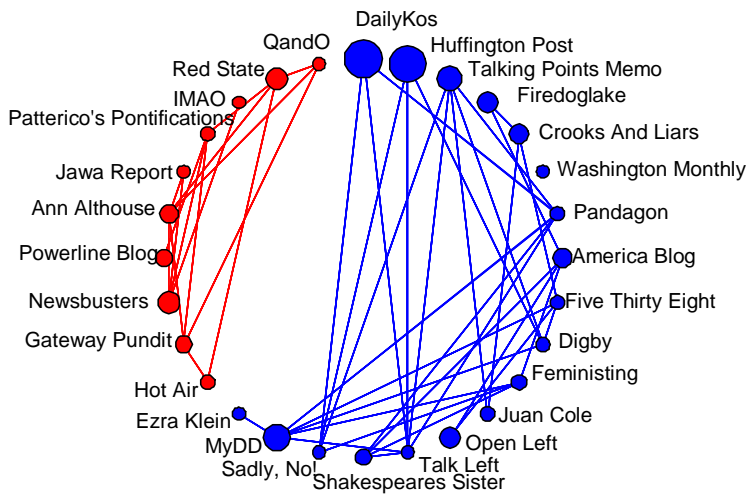

Figure 7 presents the structure of the links across political inclinations. One apparent finding is that the conservative political blogs cross-link much more to content that is linked to by liberals. According to table 2 only three consevative blogs (5\%) are isolated, meaning they link to content internally, only to videos linked to by other conservatives. Where, Liberal blogs (45) tend to segregate themeselves much more by linking to content that is linked to only by liberals $(71 \%)$.

Another apparent finding is a number of liberal top-political blogs (Digby, Juan Cole, Ezra Klein and 
Sadly, No! Shakespeares Sister) who are not referring to any content that is crossed-linked. Where only one top conservative blog is disassociated with any crossed-reference content (Hot Air).

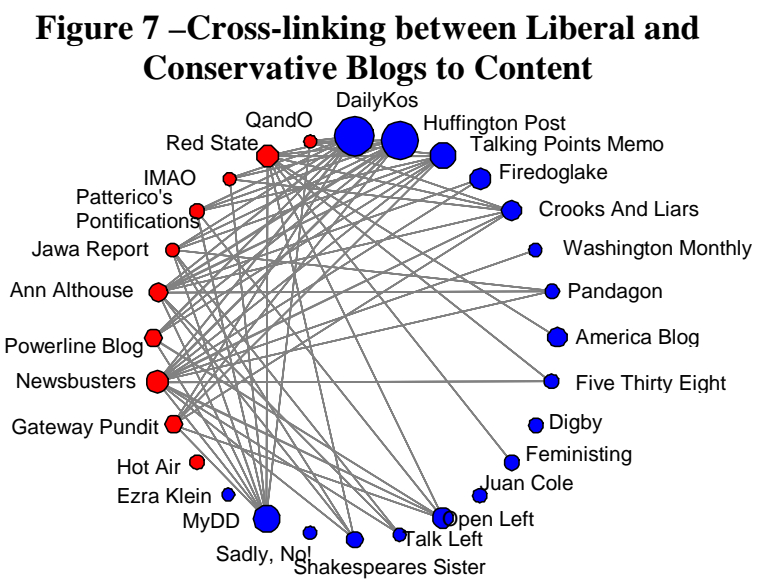

\subsection{Community as a Driver for Political Blogs}

The other two parts of our model talk about two variables which relate directly to the notion of community as a driver of participation (see figure 1). The first one is the Bandwagon effect which reflects the relationship between the popularity of a video and the tendency of the community to follow that video as well. The second variable is the number of comments which is associated in some literature as an indicator to a discursive and vibrant community [11].

Regarding the bandwagon effect, we find support for the hypothesis that a bandwagon effect exists (see table 3). The p-value is significant beyond the 0.001 level, but the effect is small. Note that since we scaled this value by 10,000 for our model, a unit change in $\mathrm{Xij}$, or 10,000 additional video views, results in a predicted increase in Yij by the value of the coefficient, or in this case, by 0.00756 . Using ratios to solve this, we find that we would expect an additional link between blogs in our network if the value of views was increased by $1,322,751$. In other words, a video that is popular with over roughly one million views is likely going to result in a bandwagon effect between blogs linking to videos (holding other factors constant). This means that popular videos have a higher likelihood to be linked to by others. Other variables such as the political inclination may weaken the bandwagon effect. We find in our model that people are more likely to respond to the political leaning rather than the bandwagon effect, which means that the political leaning has a stronger effect than the bandwagon effect. Nevertheless, the Bandwagon effect has the potential of joining blogs and bloggers from different views to the same platform.

The last variable that we examined in the context of community and blogs is the comments' effect on the participation of blogs. We fail to find support for the hypothesis that blogs with higher Community Activity Score ranking are related to the number of links a blog makes to viral videos in our set of data (see table 3). This contradicts some studies which suggest that comments are an indicator of participation [7].

\section{Discussion: homophilies and non- homophilies in multi-dimensionsional contexts and Transient Elites}

Homophily is reported in the literature of social networks and blogs [26][6]. This literature has primarily focused on links between like actors. While looking at links from actors to content, we also find political homophily, and show a stronger homophily within liberals, that is, liberal blogs tend to be associated with content that is more similar to their political inclination and view point than conservatives. This is particularly interesting in light of Benkler and Shaw's findings, that liberal blogs adopt more participatory technical platforms [13].

Do the patterns found in our paper mean that the predictions of Sunstein have come true? Has the risk of the Internet as a force that undermines deliberation and emphasizes polarization materialized? Well, not exactly.

The homophily exhibited in our paper is a monohomophily. That is, it is a homophily exhibited in one dimension among many. But we, as individuals, participate simultaneously in many dimensions. Homophily in one dimension may conflict with homophily (or non-homophily) in another. These internal conflicts may create gaps in the way we perceive ourselves, thus creating an opportunity, and demand, for deliberation within ourselves. And, this self-deliberation may later be translated and transformed into greater participation and greater discursive practices of democracy between the self and others. Castells refers to these multi-dimensions as "the sociospatial networks of power (local, 
national, global) that, in their intersection, configure societies” [2].

The structure of the network, the gatekeeping practices, and the social interactions among people all provide a context within which we may or may not exhibit homophily. Our claim is that even if a person was inclined to exhibit homophily simultaneously in all dimensions, the context and social ecology in each of those dimensions will attempt to enforce slightly different patterns of behavior on the individual - generally of a contextual mono-homophily nature appropriate for that dimension. For example, while we might exhibit a vociferous profile in blogs with people we do not know, we may behave differently in another context, like Facebook, or with our friends. Note that the claim of multi-dimensionality, on the one hand may enhance self-deliberation, it may also be interpreted as enhancing self-fragmentation of ideas.

Benkler [27] claims that the networked information economy has increased individual autonomy by increasing "the range of diversity of things that individuals can do for and by themselves". But the growing autonomy and the opportunity to be heard suggested by Benkler is often not translated into more freedoms for several reasons: users selfregulation and habits, and the interplay of network gatekeepers and gated. "The patterns of behavior emerging from behavior of the crowd, which in many cases constitute transient elites, dictate fuzziness of the notion of in/out" [17]. In other words, there are structural constraints which make a person's transformation from one dimension to another a difficult. Individuals negotiate the competing structural pressures of different dimensions within which they exist. The increased autonomy and freedom for people to discuss, and the improved ability to participate, are reflected in more alternatives. This is a good beginning, but it should also be reflected as a greater force in the holistic ecology of society. Currently, these practices represent only a minority of people.

Another important finding that needs to be discussed is that $24 \%$ of viral videos were not linked to at all by the top-blogs, and that on average each of top-blogs linked to only $13 \%$ of the viral videos. This calls for re-examination of the role of tail-blogs and top-blogs. The literature referred to in this paper focuses on top-blogs and we believe under represents the role of tail-blogs (blogs of users without high authority) in the dynamic blogosphere. The recognition of tail-blogs does not contradict patterns of the power law-distribution which have been presented in various empirical studies [2]. Also, Network Gatekeeping Theory implies a dynamic role of the gated, which may transform to network gatekeepers when exercising information control [12][28]. The blogosphere is dynamic and contextual. It is comprised of top-blogs, who are transient elites that may not act as elites in the future. Their position as elites is dependent on those they gate, and their role as the gated. In other words, contrary to off-line elites, the blogosphere elites are defined by the gated (users, tail-blogs media and political actors)-those the elite exercise information control over. The fact that many scholars agree that a power-law distribution exists indicates that the phenomenon of elites is here to stay, but the identity of these particular elites changes constantly. Additionally, the top-blogs and tail-blogs both may act as network gatekeepers with different roles. For example, while top-blogs may serve as initiators of the virality process, tail-blogs may act as distributors - or visa-versa. The important thing is to understand the transient nature of the particular elite blogs in the ecology.

Finally, while we did not examine differences in the internal structure of the actors within the blogosphere of conservatives vs. liberals like Benkler and Shaw [13], we still report meaningful differences in the behavior of conservative and liberal blogs. Our findings, that comments on sites are not a good indicator of blogs referring users to content show that scholars should be careful not to translate a high volume of discussions with actual performance or behavior. Our macro-level analysis may inform future research that can apply qualitative methods in an effort to explain why we see these patterns. The main question of whether one can translate digital activism into actual mobilization of people and bringing people to do actions remains to be seen.

\section{Conclusion}

The motivation for our study was to see how information technologies influence politics. The contribution of this study is: 1) using a hybrid theoretical framework which acknowledges homophily and the power law distribution among political blogs, and at the same time exhibits the use of the Internet also as a cross-participation platform and as strengthening participation; 2) examining empirically how top-political blogs, both liberal and conservative, refer users to content via links to viral videos during the 2008 U.S. presidential election; 3) and, we are filling a gap that exists in the literature 
which has focused mainly on the connection between blogs.

Using quantitative methods, both general descriptive statistics as well as Social Network Analysis, we analyze cases where two blogs link to the same video. We find that homophily exists within the two ideological camps; that is, liberals tend to link one set of videos, while conservatives tend to link the other. While this finding is significant, we also note that some cross-political inclination linking occurs. In these cases, the videos tend to have content that can be used for either side. We also find that a bandwagon effect exists which means that the popularity of a video (as represented by number of views) will have an effect on the likelihood that blogs will link to that popular content. Finally, we didn't find a relationship between the number of comments a blog has and the likelihood it will link to a video. Future research could examine the role of tail-blogs as opposed to top-blogs, to provide an in-depth understanding of the multiplicities of the blogosphere. Like Benkler and Shaw we believe that a qualitative examination of the patterns of behavior we analyzed is necessary.

\section{References}

[1] A. Smith, The Internet's Role in Campaign 2008, Pew Internet \& American Life Project, 2009.

[2] M. Castells, Communication power / Manuel Castells, New York: Oxford University Press, 2009.

[3] C. Sunstein, Republic.com, Princeton University Press, 2001.

[4] C. Sunstein, "Neither Hayek nor Habermas," Public Choice, vol. 134, Jan. 2008, pp. 87-95.

[5] L.A. Adamic and N. Glance, "The political blogosphere and the 2004 U.S. election: divided they blog," Proceedings of the 3rd international workshop on Link discovery, Chicago, Illinois: ACM, 2005, pp. 36-43.

[6] E. Hargittai, J. Gallo, and M. Kane, "Crossideological discussions among conservative and liberal bloggers," Public Choice, vol. 134, Jan. 2008, pp. 67-86.

[7] E. Lawrence, J. Sides, and H. Farrell, "Selfsegregation or deliberation? Blog readership, participation, and polarization in American politics,” Perspectives on Politics, vol. 8, 2010, pp. 141-157.

[8] M. Hindman, The Myth of Digital Democracy, Princeton University Press, 2008.
[9] L.A. Adamic, B.A. Huberman, A. Barabási, R. Albert, H. Jeong, and G. Bianconi, "PowerLaw Distribution of the World Wide Web," Science, vol. 287, Mar. 2000, p. 2115a.

[10] H. Farrell and D. Drezner, "The power and politics of blogs,” Public Choice, vol. 134, Jan. 2008, pp. 15-30.

[11] D. Karpf, “Understanding Blogspace,” Journal of Information Technology \& Politics, vol. 5, 2008, p. 369.

[12] K. Barzilai-Nahon, "Toward a theory of network gatekeeping: A framework for exploring information control," J. Am. Soc. Inf. Sci. Technol., vol. 59, 2008, pp. 1493-1512.

[13] Y. Benkler and A. Shaw, "A Tale of Two Blogospheres: Discursive Practices on the Left and Right."

[14] D. Woodly, "New competencies in democratic communication? Blogs, agenda setting and political participation,” Public Choice, vol. 134, Jan. 2008, pp. 109-123.

[15] L. Peer and T. Ksiazek, "What works online: Characteristics of successful videos on YouTube,” 2008.

[16] S.P. Robertson, R.K. Vatrapu, and R. Medina, "YouTube and Facebook: Online Video 'Friends' Social Networking,” 2009.

[17] K. Barzilai-Nahon, "Network Fuzziness of Inclusion-Exclusion," [forthcoming], International Journal of Communication, 2010.

[18] R.J. Klotz, "The Sidetracked 2008 YouTube Senate Campaign,” 2009.

[19] K. Wallsten, “'Yes We Can': How Online Viewership, Blog Discussion, Campaign Statements and Mainstream Media Coverage Produced a Viral Video Phenomenon,” 2009.

[20] D. Karpf, "Macaca Moments Reconsidered... YouTube Effects or Netroots Effects?,” 2009.

[21] P. Howard, "Network Ethnography and Hypermedia Organization: New Organizations, New Media, New Myths,” New Media and Society, vol. 4, 2002, pp. 550-574.

[22] H. Park and M. Thelwall, "The network approach to web hyperlink research and its utility for science communication," Virtual methods: issues in social research on the Internet, C. Hine, Ed., Berg, 2005, pp. 171-81.

[23] D. Karpf, Measuring Influence in the Political Blogosphere: Who is Winning and How Can We Tell?, George Washigton Univeristy's Institute for Politics, Democracy and the Internet, 2008.

[24] C. Butts, "Social network analysis: A methodological introduction," Asian Journal of Social Psychology, vol. 11, 2008, p. 13. 
[25] M. Morris, C. Butts, D. Hunter, M. Handcock, and S. Goodreau, “A statnet Tutorial,” Journal of Statistical Software, vol. 24, 2008.

[26] M. McPherson, L. Smith-Lovin, and J.M. Cook, "Birds of a Feather: Homophily in Social Networks," Annual Review of Sociology, vol. 27, 2001, pp. 415-444.

[27] Y. Benkler, The Wealth of Networks: How Social Production Transforms Markets and Freedom, Yale University Press, 2007.

[28] K. Barzilai-Nahon, "Gatekeeping: A Critical Review," Annual Review of Information Science and Technology, vol. 43, 2009, pp. 433-478. 